\title{
DISCOVERY OF AN EXTENDED DEBRIS DISK AROUND THE F2 V STAR HD 15745
}

\author{
Paul Kalas, ${ }^{1,2}$ Gaspard Duchene, ${ }^{1,3}$ Michael P. Fitzgerald, ${ }^{2,4,5}$ and James R. Graham ${ }^{1,2}$ \\ Received 2007 September 12; accepted 2007 November 2; published 2007 November 26
}

\begin{abstract}
Using the Advanced Camera for Surveys aboard the Hubble Space Telescope, we have discovered dust-scattered light from the debris disk surrounding the F2 V star HD 15745. The circumstellar disk is detected between 2.0" and $7.5^{\prime \prime}$ radius, corresponding to $128-480 \mathrm{AU}$ radius. The circumstellar disk morphology is asymmetric about the star, resembling a fan, and consistent with forward scattering grains in an optically thin disk with an inclination of $\sim 67^{\circ}$ to our line of sight. The spectral energy distribution and scattered light morphology can be approximated with a model disk composed of silicate grains between 60 and 450 AU radius, with a total dust mass of $10^{-7} M_{\odot}\left(0.03 M_{\oplus}\right)$, representing a narrow grain size distribution $(1-10 \mu \mathrm{m})$. Galactic space motions are similar to the Castor moving group, with an age of $\sim 10^{8} \mathrm{yr}$, although future work is required to determine the age of HD 15745 using other indicators.
\end{abstract}

Subject headings: circumstellar matter — stars: individual (HD 15745)

\section{INTRODUCTION}

The collisional debris from solid bodies orbiting main-sequence stars results in circumstellar dust disks that produce excess emission at thermal infrared wavelengths (Backman \& Paresce 1993). Excess thermal emission may also arise from a star heating interstellar dust (e.g., Kalas et al. 2002); therefore, obtaining images of dust-scattered light is a key method for constraining the origin of dust. Furthermore, direct images of dust-scattered light may be used to obtain the color and polarization properties of the grains, which depend on their size distribution, composition, and structure (e.g., Graham et al. 2007). The overall geometry of a debris disk may also reveal sources of dynamical perturbations, such as those from stellar and substellar companions (e.g., Roques et al. 1994; Liou \& Zook 1999; Moro-Martin \& Malhotra 2002).

Here we show the first scattered-light images of a debris disk surrounding HD 15745, an F2 V star at 64 pc. Silverstone (2000) first reported HD 15745 as a debris disk candidate star based on excess thermal emission $L_{\mathrm{IR}} / L_{\star}=1.8 \times 10^{-3}$ using IRAS data, and $L_{\mathrm{IR}} / L_{\star}=1.3 \times 10^{-3}$ using ISO data. Zuckerman \& Song (2004) modeled the spectral energy distribution (SED) with a single-temperature dust belt at $27 \mathrm{AU}$ radius with $L_{\mathrm{IR}} / L_{\star}=1.2 \times 10^{-3}$.

\section{OBSERVATIONS \& DATA ANALYSIS}

We observed HD 15745 on 2006 July 17 with the Hubble Space Telescope (HST) Advanced Camera for Surveys (ACS) High Resolution Channel (HRC) coronagraph. The star was acquired behind the $1.8^{\prime \prime}$ diameter occulting spot, and three $700 \mathrm{~s}$ integrations with the F606W $\left(\lambda_{c}=591 \mathrm{~nm}, \Delta \lambda=234 \mathrm{~nm}\right)$ broadband filter were obtained at a fixed position angle. The three frames were median combined for cosmic-ray rejection. The point-spread function (PSF) was then subtracted using images from the $H S T$ archive of five $\mathrm{F}$ stars obtained with the

\footnotetext{
${ }^{1}$ Astronomy Department and Radio Astronomy Laboratory, University of California, Berkeley, CA.

${ }^{2}$ National Science Foundation Center for Adaptive Optics, University of California, Santa Cruz, CA.

${ }^{3}$ Laboratoire d'Astrophysique de Grenoble, Université Joseph Fourier, Grenoble Cedex 9, France.

${ }^{4}$ Lawrence Livermore National Laboratory, Livermore, CA.

${ }^{5}$ Michelson Fellow.
}

ACS coronagraph at the same wavelength and comparable integration times. All five versions of a PSF-subtracted image of HD 15745 show the fan-shaped circumstellar nebulosity. The five versions were then median combined to produce a final image that was then corrected for geometric distortion. We also detected HD 15745 from Gemini North with the Michelle camera on 2006 September 20, using the $N^{\prime}$ filter. We measure a flux of $N^{\prime}=74.7 \pm 2.9$ mJy within a $1.4^{\prime \prime}$ radius aperture, with an aperture correction based on near-contemporaneous images of the photometric standard star (HD 20893).

\section{RESULTS}

The PSF-subtracted HST data reveal a fan-shaped nebulosity surrounding HD 15745 in the approximate region between P.A. $=190^{\circ}$ and $10^{\circ}$ (Fig. 1). We interpret the nebulosity as an azimuthally symmetric circumstellar disk inclined to our line of sight and composed of dust that has an asymmetric scattering phase function that produces a fanlike appearance (Kalas et al. 2006). Another possibility, asymmetric grain erosion and blowback by encounters with the interstellar medium (Artymowicz \& Clampin 1997), is less likely, given the southeastward proper motion $\left(\mu_{\alpha}=46\right.$ mas $\mathrm{yr}^{-1}, \mu_{\delta}=-47$ mas $\left.\mathrm{yr}^{-1}\right)$ and location of the star well within the local interstellar bubble (Kalas et al. 2002), although at least one exception has been found for the latter point (Gáspár et al. 2007).

In principle, the detection limit for the inner radius of the nebulosity is set by the occulting spot radius $\left(0.9^{\prime \prime}\right)$, but the presence of quasi-static speckles produces significant PSF subtraction artifacts, limiting the inner detection radius to $\sim 2$ " (128 $\mathrm{AU})$ radius. The outer radius of the disk is a sensitivity-limited value. In data that have been binned $8 \times 8$ pixels $\left(0.2^{\prime \prime} \times\right.$ $\left.0.2^{\prime \prime}\right)$, the disk is detected as far as $\sim 7.5^{\prime \prime}(480 \mathrm{AU}$ ) radius. The 23.0 mag $\operatorname{arcsec}^{-2}$ surface brightness isophote (Fig. 2) shows a relatively symmetric fanlike morphology. The radial surface brightness profiles (Fig. 3) are monotonic and can be approximated by power laws with an index of $-3.3 \pm 0.1$.

\section{DISK MODELS}

We model the SED and scattered light appearance of the HD 15745 disk using a Monte Carlo radiation transfer code (MCFOST; Pinte et al. 2006) in which a star radiates isotropically in space and illuminates an azimuthally symmetric parameterized 


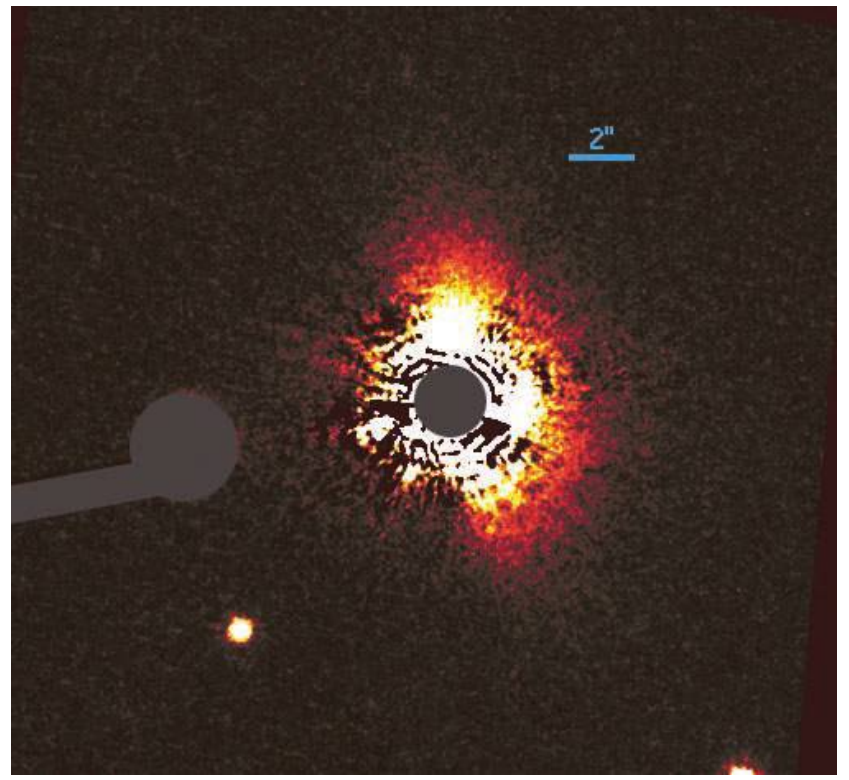

FIG. 1.-False-color, PSF-subtracted image of HD 15745. North is up, east is left. Observations were made using the F606W filter and the ACS HRC. Gray fields cover the focal-plane masks in the ACS HRC. The axis of symmetry for the nebulosity is P.A. $\simeq 280^{\circ}$.

disk. In a first step, we determine the stellar parameters by fitting the SED up to $11 \mu \mathrm{m}$ (Fig. 4). Using NextGen models (Baraffe et al. 1998) for the stellar photosphere, we find that the best-fit model has $T_{\text {eff }}=6800 \mathrm{~K}$ and $R_{\star}=1.31 R_{\odot}$.

We then proceed to fit the entire SED of HD 15745 to determine the disk properties. In order to consider as few free parameters as possible, we adopt a $\rho(r, z) \propto r^{\alpha} \exp \left(-z^{2} / 2 \sigma_{z}^{2}\right)$ number density distribution, with $\alpha=-1$ and a constant

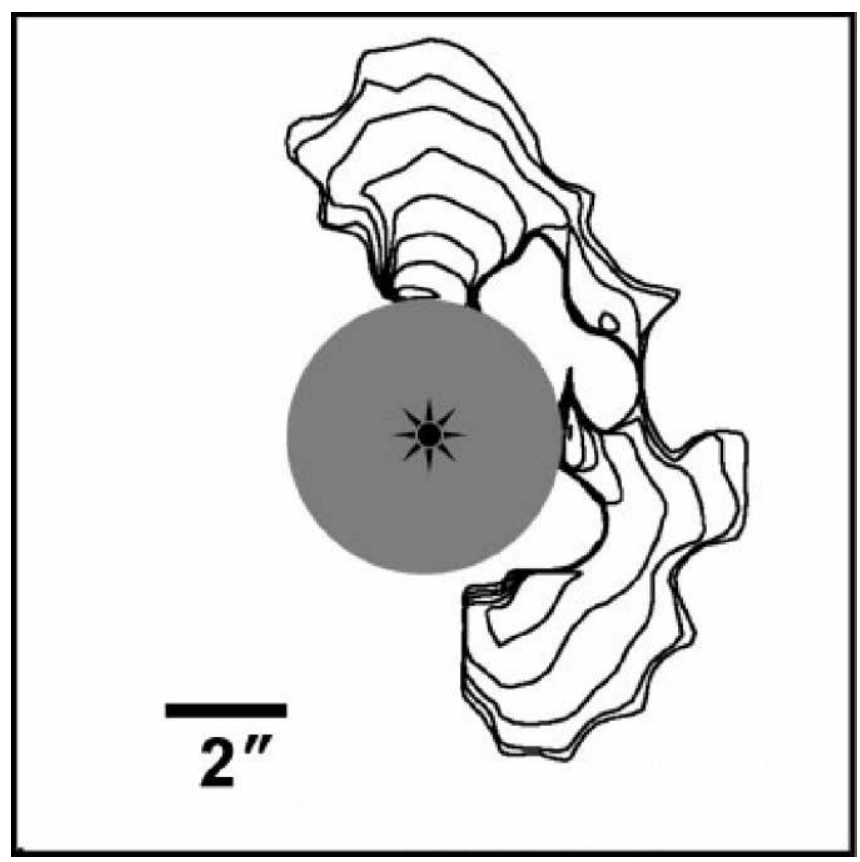

FIG. 2.-Surface brightness isocontours for the HD 15745 debris disk converted from F606W to Johnson $V$-band (derived using STSDAS/SYNPHOT with a Kurucz model atmosphere and the appropriate observatory parameters). North is up and east is left. The outermost contour is $23.0 \mathrm{mag} \operatorname{arcsec}^{-2}$, with a contour interval of $0.5 \mathrm{mag} \operatorname{arcsec}^{-2}$.

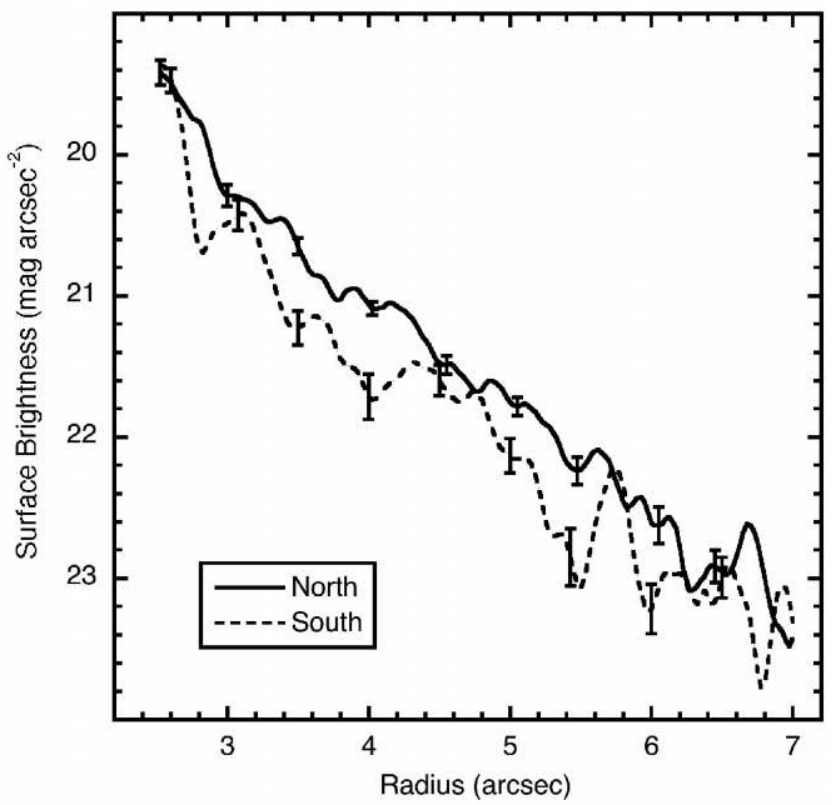

FIG. 3.- $V$-band radial surface brightness cuts between $2.5^{\prime \prime}$ and $7.0^{\prime \prime}$ radius along the northern (P.A. $=360^{\circ}$ ) and southern $\left(\right.$ P.A. $=210^{\circ}$ ) portions of the HD 15745 nebulosity. We extracted photometry in boxes with sizes of $0.1^{\prime \prime}$ (along the radial direction) by $0.5^{\prime \prime}$, and we plot the median value from the five subtractions of the HD 15745 PSF. Representative error bars show the standard deviation of these five values for each box. The noise due to PSF subtraction artifacts is greater for the south side of the disk, as evidenced by the rippled form of the radial profile on scales of a few tenths of an arcsecond and the larger error bars. In some radial regions, the south side of the disk may be fainter than the north side by $\sim 0.4 \mathrm{mag} \operatorname{arcsec}^{-2}$, but these surface brightness asymmetries should be verified in future data that have higher signalto-noise ratio.

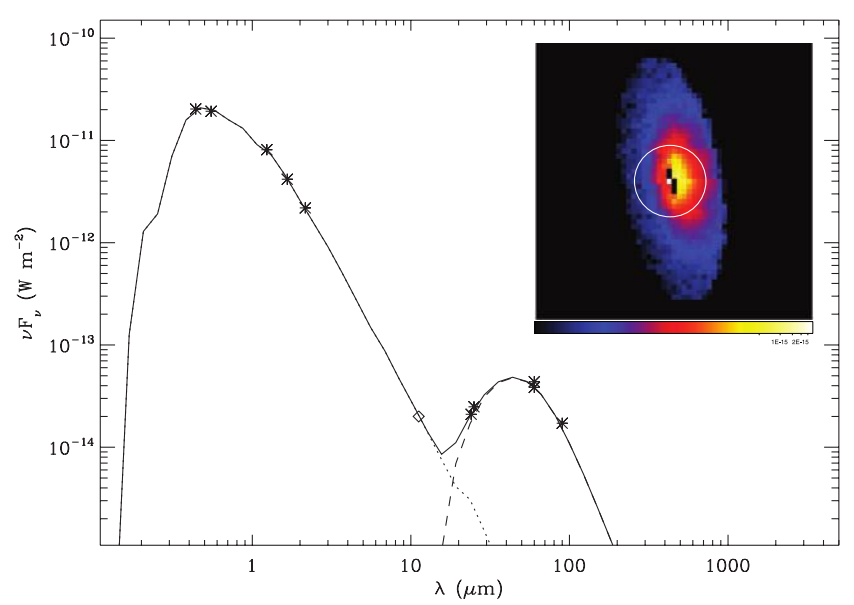

FIG. 4.-Observed SED of HD 15745 along with our best-fitting model (see text for model parameters). Our new $N^{\prime}\left(\lambda_{c}=11.2 \mu \mathrm{m}, \Delta \lambda=2.4 \mu \mathrm{m}\right)$ Gemini measurement is indicated with a diamond (Gemini program ID GN-2006B-C12). HD 15745 is unresolved, with a FWHM of $0.3^{\prime \prime}$ in the north-south direction, and $0.5^{\prime \prime}$ in the east-west direction. Photometric calibration was accomplished by observing HD 20893 in $N^{\prime}$ and assuming an extinction of $0.172 \mathrm{mag}$ per air mass. The $24-90 \mu \mathrm{m}$ data represent the IRAS and $I S O$ values given by Moór et al. (2006). The dotted and dashed curves represent the stellar and disk emission, respectively. The inset is a log-stretch image of our bestfit model, observed with an inclination of $67^{\circ}$ and rotated so as to match the orientation of the disk in Fig. 1. The white circle represents the 2 " radius region that is hidden in Figs. 1 and 2. 
$\sigma_{z}=2 \mathrm{AU}$, that extends from $R_{\text {in }}$ to $R_{\text {out }}=450 \mathrm{AU}$ and sums up to a total disk mass $M_{d}$. The choice of $\sigma_{z}=2 \mathrm{AU}$ is consistent with disk heights inferred from the edge-on debris disks $\beta$ Pic and AU Mic (Kalas \& Jewitt 1995; Krist et al. 2005) and does not significantly influence our results for values $\sigma_{z} \geq 1 \mathrm{AU}$ (which is required to keep the disk optically thin to starlight). The $r^{-1}$ number density profile fits the observed slope of the surface brightness (see below). We also fix the dust grain properties to be a collection of compact spherical silicate grains from Draine \& Lee (1984), with size distribution $N(a) \propto$ $a^{-3.5}$ from $a_{\min }$ to $a_{\max }=10 a_{\min }$. Because the disk is assumed to be gas-free, each grain size has its own temperature: smaller grains are hotter than larger at a given distance from the star. Therefore, for each value of $a_{\text {min }}$, we need to adjust $R_{\text {in }}$ to obtain a maximum dust temperature in the range $60-80 \mathrm{~K}$; i.e., a peak dust emission around $40-50 \mu \mathrm{m}$ (Fig. 4). Once this is set, we can adjust the total dust mass to fit the SED. For values of $a_{\min }$ ranging from 0.125 to $8 \mu \mathrm{m}$ (with a factor of 2 increment), we perform this two-parameter adjustment using a grid of nine regularly sampled values of $R_{\text {in }}$ and $M_{d}$ and compute the associated $\chi^{2}$ value. The narrowness of the disk thermal emission peak requires $a_{\max } \lesssim 10 \mu \mathrm{m}$ (hence $a_{\min } \lesssim 1 \mu \mathrm{m}$ ). Larger grains deviate from the Rayleigh regime in the midinfrared and result in a much broader emission peak than is observed. By allowing a smaller value of $a_{\min }$ and larger values of $a_{\text {max }} / a_{\text {min }}$, we do not find a lower limit to $a_{\text {min }}$ from the analysis of the SED alone, down to at least $0.1 \mu \mathrm{m}$. Provided $R_{\text {in }}$ and $M_{d}$ are adjusted accordingly, we always find satisfying fits to the SED.

We then explore the disk surface brightness in order to better constrain $a_{\text {min }}$. Indeed, assuming $a_{\max } / a_{\text {min }}=10$, the scattering properties of the dust populations considered here vary strongly with $a_{\text {min }}$. The albedo at $0.606 \mu \mathrm{m}$ decreases from 0.82 to 0.65 as $a_{\text {min }}$ increases from 0.125 to $1 \mu \mathrm{m}$, because of the depletion of the highly reflective small grains. At the same time, the scattering asymmetry parameter, $g=\langle\cos \theta\rangle$, increases from 0.65 to 0.89 because large grains scatter preferentially forward. As a consequence, the models with small values of $a_{\min }$ can be rejected based on our ACS image: simulated images of the disk with $a_{\text {min }}=0.125 \mu \mathrm{m}$ are $\sim 4 \mathrm{mag} \operatorname{arcsec}^{-2}$ too bright compared to our observations. Furthermore, with such a dust population, the predicted flux ratio between the front and back side is low enough, independently of the inclination, that it should have been detected in our data set. To fit both the surface brightness of the disk and the apparent front/back asymmetry, our model indicates that $a_{\text {min }}>0.5 \mu \mathrm{m}$, implying a narrow grain size distribution and justifying a posteriori our assumption of $a_{\text {max }} / a_{\text {min }}=10$.

Figure 4 illustrates our best-fitting model, which has $a_{\text {min }}=1 \mu \mathrm{m}, a_{\max }=10 \mu \mathrm{m}, R_{\text {in }}=60 \mathrm{AU}, M_{d}=10^{-7} M_{\odot}$, and $L_{\mathrm{IR}} / L_{\star}=2.2 \times 10^{-3}$. Assuming a disk inclination $i=$ $65^{\circ}-70^{\circ}$, a power-law fit to the modeled surface brightness profiles results in a $\sim-3.5$ index, and the observed front/back intensity ratio at $2.3^{\prime \prime}$ along the disk semiminor axis is $\gtrsim 10$. Both figures are consistent with the observations. Subsequent models were calculated with $a_{\min }=0.75$ and $1.25 \mu \mathrm{m}$ to better constrain this parameter. We obtained marginally acceptable surface brightness fits in both cases, and we conclude that $0.75 \mu \mathrm{m} \leq a_{\min } \leq 1.25 \mu \mathrm{m}$ in the disk surrounding HD 15745 . This result is consistent with a grain blowout size of $\sim 1 \mu \mathrm{m}$ calculated for silicate grains. Associated uncertainties on the disk inner radius and total disk mass are on the order of $10 \%$. Our quantitative estimates of $a_{\min }$ and $a_{\max }$ depend on the assumptions we made, but the overall conclusion (order of mag- nitudes for grain sizes, hence inner radius and total dust mass, and the need for a narrow size distribution) are robust.

\section{DISCUSSION}

HD 15745 is now the fifth main-sequence F star with a debris disk resolved in scattered light. Three of these have ringlike structure, whereas HD 15745 shares a wide-disk architecture with HD 15115 (F2 V; Kalas et al. 2007). The three ringlike architectures are found around HD 139664 (F5 V; Kalas et al. 2006), HD 181327 (F5/F6; Schneider et al. 2006), and HD 10647 (F9 V; Stapelfeldt et al. 2007). At the present time, the dust properties have been modeled only for HD 181327, with a Henyey-Greenstein scattering asymmetry parameter $g=$ $0.3 \pm 0.03$ (Schneider et al. 2006). For debris disks around stars of other spectral types, AU Mic (M1 V) has a scattering asymmetry parameter similar to that of HD 15745 (Graham et al. 2007), whereas most other debris disk, such as Fomalhaut (A3 V; Kalas et al. 2005) and HD 107146 (G5 V; Ardila et al. 2004) have a low scattering asymmetry, comparable to that of HD 181327. Our present model suggests a concentration of dust grains in the range $1-10 \mu \mathrm{m}$, which is consistent with a wavy grain size distribution (departure from a simple power law; e.g., Campo Bagatin et al. 1994; Thebault \& Augereau 2007). Small grains do not exist in sufficient quantity to counteract the highly asymmetric scattering phase function of the larger grains, whereas in other debris disk systems the collisional cascade may be robust to the smallest sizes, producing relatively symmetric scattering. Further refinements for the properties of dust around HD 15745 and other debris disks requires resolved imaging observations at other wavelengths and with polarization. Ultimately, the explanation for why one debris disk has a particular grain size distribution may depend on differences in disk dynamical properties and the composition of grains (e.g., volatile vs. refractory elements).

Among the F stars discussed above, HD 15115 and HD 181327 may belong to the $\beta$ Pic moving group, with ages of 12 Myr (Zuckerman et al. 2001). HD 15745, on the other hand, may be a member of the Castor moving group, based on its Galactic space motion. Moór et al. (2006) calculate $(U, V, W)=(-16.5 \pm 1.1,-10.8 \pm 1.3,-10.7 \pm 0.7) \mathrm{km}$ $\mathrm{s}^{-1}$ for HD 15745, consistent with $(U, V, W)=(-10.7 \pm 3.5$, $-8.0 \pm 2.4,-9.7 \pm 0.7) \mathrm{km} \mathrm{s}^{-1}$ given for the Castor moving group (CMG; Barrado y Navascues 1998). Among the CMG members, HD 38678 ( $\zeta$ Lep), another well-known debris disk star, has a Galactic space velocity $(U, V, W)=(-14.4 \pm 3.6$, $-11.1 \pm 3.0,-8.4 \pm 1.8) \mathrm{km} \mathrm{s}^{-1}$ that is closest to that of HD 15745. Barrado y Navascues (1998) estimate that the CMG has an age of $200 \pm 100 \mathrm{Myr}$, although Zuckerman \& Song (2004) estimate a younger age for HD 15745 (“30?” Myr), based on its location on an A-star H-R diagram and the relatively large dust optical depth. Moór et al. (2006) similarly conclude that nearly all stars with $L_{\mathrm{IR}} / L_{\star}>5 \times 10^{-4}$ are younger than $10^{8} \mathrm{yr}$. The infrared excess for HD 15745 is an order of magnitude greater than that of CMG members Fomalhaut, Vega, and $\zeta$ Lep. From the five CMG candidates that are $\mathrm{F}$ stars, two have been detected at far-infrared wavelengths. HD $119124(\mathrm{~F} 7.7 \mathrm{~V})$ has $L_{\mathrm{IR}} / L_{\star}=(3-6) \times 10^{-5}$, comparable to that of Fomalhaut and Vega (Trilling et al. 2007). HD 130819 (F3 V) has $L_{\mathrm{IR}} / L_{\star}=3 \times 10^{-4}$ (Chen et al. 2005), which is still a factor of 7 less than that of HD 15745 .

Recent work demonstrates a large spread in dust optical depths at a given age (Rhee et al. 2007), which may be a consequence of the spread of initial disk masses (Wyatt et al. 
2007). Therefore, the high optical depth of HD 15745 cannot uniquely discriminate between a $\sim 10 \mathrm{Myr}$ or a $100 \mathrm{Myr}$ age. Assuming steady-state evolution, the dust optical depth of HD 15745 could arise from a 10 Myr old debris disk that started with $\sim 10 M_{\oplus}$ of solid material, or a 100 Myr old disk that started with $\sim 100 M_{\oplus}$ of solid material. The ambiguity is augmented by allowing stochastic spikes in dust production that may occur in a few percent of debris disks. (e.g., Beichman et al. 2006). A more precise age for HD 15745 therefore requires further input using stellar properties. However, we note that the large geometric vertical optical depth of our model $(\tau=$ $5.8 \times 10^{-3}$ at $60 \mathrm{AU}$ radius) implies a fiducial collisional destruction timescale $t_{c} \sim 1 / \Omega \tau \sim 10^{5} \mathrm{yr}$ at $60 \mathrm{AU}$. This is significantly shorter than the age estimates for HD 15745, implying that the circumstellar dust is collisionally evolved. Moreover, the rapid collision timescale indicates that the radius of the inner disk hole approximates the location of the planetesimal belt that regenerates the observed dust, instead of signifying the radius where grains inspiraling from PoyntingRobertson drag are trapped in resonances with planetary bodies (Wyatt 2005).

\section{SUMMARY}

Using the HST ACS coronagraph, we present the first images of dust-scattered light from a debris disk surrounding the F2 V star HD 15745. The fan-shaped morphology is consistent with an azimuthally symmetric disk with asymmetric isophotes due to an asymmetric dust scattering phase function combined with a $67^{\circ}$ inclination to the line of sight. HD 15745 belongs in a category of wide disks with a sensitivity-limited outer extent near 500 AU. Our silicate-grain models for the SED and the disk surface brightness suggest an inner disk radius of $60 \mathrm{AU}$, a somewhat narrow grain size distribution between 1 and $10 \mu \mathrm{m}$, and a total disk mass of $10^{-7} M_{\odot}$. Future observations should determine the optical and near-infrared colors, as well as polarization, to further constrain grain properties. High-resolution imaging of the inner part of the disk should reveal the inner hole that in the model presented here has radius of $60 \mathrm{AU}$, or $0.5^{\prime \prime}$ radius from the star projected on the sky. Due to the asymmetric scattering phase function, the inner boundary of the disk hole should appear as a $1^{\prime \prime} \times 2^{\prime \prime}$ elliptical feature. Our silicate-grain disk model predicts a peak $V$-band and $H$-band surface brightnesses of 14.8 and $12.7 \mathrm{mag} \mathrm{arcsec}^{-2}$, respectively, or about 1 mag redder than the star itself. Nearinfrared data would thereby test our present disk model that is dominated by relatively large grains $(>1 \mu \mathrm{m})$, where the albedo is not significantly diminished in the near-infrared, yet the nearinfrared scattering is more isotropic ( $g=0.57$ in $H$ vs. 0.89 in $V$ ) as the observing wavelength approaches the grain size.

Support for GO-10896 was provided by NASA through a grant from STScI under NASA contract NAS 5-26555. We used the Gemini Observatory, operated by AURA, under agreement with the NSF. This work was supported by the NSF Science and Technology Center for Adaptive Optics, managed by UCSC under agreement AST 98-76783, and by the Michelson Fellowship Program.

\section{REFERENCES}

Ardila, D. R., et al., 2004, ApJ, 617, L147

Artymowicz, P., \& Clampin, M. 1997, AJ, 490, 863

Backman, D. E., \& Paresce, F. 1993, in Protostars and Planets III, ed. E. H. Levy \& J. I. Lunine (Tucson: Univ. Arizona Press), 1253

Baraffe, I., Chabrier, G., Allard, F., \& Hauschildt, P. H. 1998, A\&A, 337, 403

Barrado y Navascues, D. 1998, A\&A, 339, 831

Beichman, C. A., et al. 2006, ApJ, 639, 1166

Campo Bagatin, A., Cellino, A., Davis, D. R., Farinella, P., \& Paolicchi, P. 1994, Planet. Space Sci., 42, 1079

Chen, C. H., et al. 2005, ApJ, 634, 1372

Draine, B. T., \& Lee, H. M. 1984, ApJ, 285, 89

Gáspár, A., Su, K. Y. L., Rieke, G. H., Balog, Z., Kamp, I., Martínez-Galarza, J. R., \& Stapelfeldt, K. 2007, ApJ, in press

Graham, J. R., Kalas, P., \& Matthews, B. 2007, ApJ, 654, 595

Kalas, P., Fitzgerald, M. P., \& Graham, J. R. 2007, ApJ, 661, L85

Kalas, P., Graham, J. R., Beckwith, S. V. W., Jewitt, D. C., \& Lloyd, J. P. 2002, ApJ, 567, 999

Kalas, P., Graham. J. R., \& Clampin, M. C. 2005, Nature, 435, 1067

Kalas, P., Graham. J. R., Clampin, M. C., \& Fitzgerald, M. P. 2006, ApJ, 637, L57
Kalas, P., \& Jewitt, D. 1995, AJ,110, 794

Krist, J. E., et al. 2005, ApJ, 129, 1008

Liou, J.-C., \& Zook, H. A. 1999, AJ, 118, 580

Moór, A., Abraham, A., \& Derekas, A. 2006, ApJ, 644, 525

Moro-Martin, A., \& Malhotra, R. 2002, AJ, 124, 2305

Pinte, C., Menard, F., Duchene, G., \& Bastien, P. 2006, A\&A, 459, 797

Rhee, J. H., Song, I., Zuckerman, B., \& McElwain, M. 2007, ApJ, 660, 1556

Roques, F., Scholl, H., Sicardy, B., \& Smith, B. A. 1994, Icarus, 108, 37

Schneider, G., et al. 2006, ApJ, 650, 414

Silverstone, M. D. 2000, Ph.D. thesis

Stapelfeldt, K. R., Krist, J., Bryden, G., \& Chen, C. 2007, in In the Spirit of Bernard Lyot: The Direct Detection of Planets and Circumstellar Disks in the 21st Century, ed. Paul Kalas (Berkeley: Univ. of California), 47

Thebault, P., \& Augereau, J.-C. 2007, A\&A, 472, 169

Trilling, D. E., et al. 2007, ApJ, 658, 1289

Wyatt, M. C. 2005, A\&A, 433, 1007

Wyatt, M. C., et al. 2007, ApJ, 663, 365

Zuckerman, B., Song, I., Bessel, M. S., \& Webb, R. A. 2001, ApJ, 562, L87

Zuckerman, B., \& Song, I. 2004, ApJ, 603, 738 